\title{
Natural Course of Dissecting Vertebrobasilar Artery Aneurysms without Stroke
}

\author{
N. Kobayashi, Y. Murayama, I. Yuki, T. Ishibashi, M. Ebara, H. Arakawa, K. Irie, H. Takao, I. Kajiwara,
}

K. Nishimura, K. Karagiozov, and M. Urashima

\begin{abstract}
BACKGROUND AND PURPOSE: The natural history and therapeutic management of dissecting vertebrobasilar aneurysms without ischemic or hemorrhagic stroke (nonstroke dissecting vertebrobasilar aneurysms) are not well-established. We conservatively followed patients with nonstroke dissecting vertebrobasilar aneurysms and evaluated the factors related to clinical and morphologic deterioration.
\end{abstract}

MATERIALS AND METHODS: One hundred thirteen patients were enrolled and divided by clinical presentation at diagnosis: asymptomatic (group 1, $n=52$ ), pain only (group 2, $n=56$ ), and mass effect (group 3, $n=5$ ). Patients were conservatively managed without intervention and antithrombotic therapy. Clinical outcomes and morphologic changes were analyzed.

RESULTS: A total of 113 patients who were diagnosed with nonstroke dissecting vertebrobasilar aneurysm had a mean follow-up of 2.9 years (range, 27 days to 8 years). Throughout that period, 1 patient in group 1 (1.9\%) and 1 patient in group 2 (1.8\%) showed clinical deterioration due to mass effect, and 1 patient in group $3(20 \%)$ developed ischemic stroke followed by subarachnoid hemorrhage. Most patients (97.3\%) were clinically unchanged. Three patients who had clinical deterioration showed aneurysm enlargement $(P<.001)$. Aneurysms remained morphologically unchanged in 91 patients (80.5\%). Aneurysm enlargement was seen in 5 patients (4.4\%); risk of enlargement was significantly associated with either maximum diameter (hazard ratio $=1.30 ; 95 \% \mathrm{Cl}, 1.11-11.52 ; P=.001$ ) or aneurysm $\geq 10$ $\mathrm{mm}$ (hazard ratio $=18.0 ; 95 \% \mathrm{Cl}, 1.95-167 ; P=.011)$.

CONCLUSIONS: The natural course of these lesions suggests that acute intervention is not always required and close follow-up without antithrombotic therapy is reasonable. Patients with symptoms due to mass effect or aneurysms of $>10$ mm may require treatment.

ABBREVIATIONS: DVBA = dissecting vertebrobasilar aneurysm

T he detection and diagnosis of dissecting vertebrobasilar aneurysms without ischemic or hemorrhagic stroke (nonstroke DVBAs) have increased due to recent advancements in noninvasive brain imaging screening techniques. At the same time, the natural history of nonstroke DVBA is unknown. ${ }^{1,2}$ Furthermore, the risks and benefits of antiplatelet or anticoagulation therapy for nonstroke DVBA are unclear. The aim of this study was to evaluate the natural course of nonstroke DVBA by identifying the factors associated with clinical and morphologic deterioration and thus contributing to the optimal management strategy.

Received August 30, 2013; accepted after revision December 2.

From the Department of Neurosurgery (N.K., Y.M., I.Y., T.I., M.E., H.A., K.I., H.T., I.K., K.N., K.K.) and Division of Molecular Epidemiology (M.U.), The Jikei University School of Medicine, Tokyo, Japan.

Please address correspondence to Yuichi Murayama, MD, Department of Neurosurgery, The Jikei University School of Medicine, 3-25-8 Nishi-Shinbashi, Minato-ku, Tokyo, Japan; e-mail: ymurayama@jikei.ac.jp

http://dx.doi.org/10.3174/ajnr.A3873

\section{MATERIALS AND METHODS}

From October 2003 to April 2012, one hundred fifty-five patients at our institution were diagnosed with intracranial DVBA, defined as a nonsaccular aneurysm located at a nonbranching site of the vertebrobasilar artery system. Patients with subarachnoid hemorrhage $(n=11)$, ischemic stroke $(n=8)$, prior surgery $(n=$ $11)$, and a single visit for a second opinion $(n=12)$ were excluded; thus, 113 patients were enrolled prospectively in this study. It was approved by the institutional review board of our university, and informed consent was obtained from all patients for inclusion.

The 113 patients were divided into 3 groups based on their clinical presentation at diagnosis. Group 1 included asymptomatic patients who were diagnosed incidentally on a prophylactic brain screening MRA study $(n=52)$, group 2 patients presented with pain only $(n=56)$, and group 3 patients presented with mass effect from the aneurysm $(n=5)$. A history of pain was considered relevant only when the patient had a sudden onset of neck or back pain and the date and timing were clearly indicated (Table 1). Patients were followed conservatively with noninvasive imag- 
ing studies, without endovascular or surgical intervention. The existence of pre-existing conditions and risk factors, such as hypertension, hyperlipidemia, and current smoking, was evaluated; however, antiplatelet or anticoagulation therapy was not applied in addition to their pre-existing management.

Radiographic evaluation was performed by using 3D-CTA (Somatom Sensation 16; Siemens, Erlangen, Germany) according to our currently accepted protocol. The parameters include FOV $=180,100 \mathrm{kV}, 250 \mathrm{~mA}, 60-\mathrm{mL}$ contrast at $4 \mathrm{~mL} / \mathrm{s}$. The data were transferred to a 3D workstation (TeraRecon, San Mateo, California) by using stretch view software, and the maximum diameter of each aneurysm was measured (including the parent artery, $n=92$ ) (Fig 1). Patients who had contraindications or refused contrast-enhanced CTA $(n=21)$ were evaluated by MR

angiography (Magnetom Symphony 1.5T; Siemens), and their aneurysms were measured by using axial source images from time-of-flight sequences. All 113 patients were observed with consecutive 3D-CTA and/or MRA TOF. Morphologic evaluation classified results as "unchanged," "improved," or "enlarged." "Improved" was defined as any reduction by $\geq 1 \mathrm{~mm}$ in aneurysm diameter or normalization of pearl-and-strings findings (ie, stenosis normalization).

Clinical outcomes were evaluated with the modified Rankin Scale, and "deteriorated" was defined as $\geq 1$ point deterioration on the scale.

To find the factors related to clinical and morphologic changes, we also included the following criteria: sex, size $(<10$ $\mathrm{mm}$ versus $>10 \mathrm{~mm}$ ), initial presentation, pre-existing diseases, and smoking history.

Table 1: Patient characteristics stratified by clinical presentation at diagnosis

\begin{tabular}{|c|c|c|c|c|}
\hline & $\begin{array}{c}\text { Group 1: } \\
\text { Asymptomatic } \\
(n=52)\end{array}$ & $\begin{array}{l}\text { Group 2: } \\
\text { Pain } \\
(n=56)\end{array}$ & $\begin{array}{c}\text { Group 3: } \\
\text { Mass Effects } \\
(n=5)\end{array}$ & $P$ Value \\
\hline Age (yr) (mean) (SD) ${ }^{a}$ & $59.3(9.4)$ & $51.1(10.6)$ & $54.0(11.7)$ & $.0002^{\mathrm{a}}$ \\
\hline Male (No.) (\%) & $38(73.1)$ & $44(78.6)$ & $4(80.0)$ & $.78^{\mathrm{b}}$ \\
\hline Hypertension (No.) (\%) & $35(67.3)$ & 19 (33.9) & $2(40.0)$ & $.002^{b}$ \\
\hline Diabetes (No.) (\%) & $1(1.9)$ & $5(8.9)$ & $0(0.0)$ & $.23^{\mathrm{b}}$ \\
\hline Hyperlipidemia (No.) (\%) & $7(13.5)$ & $14(25.0)$ & $2(40.0)$ & $.18^{\mathrm{b}}$ \\
\hline Current smoker (No.) (\%) & $24(46.2)$ & $26(46.4)$ & $1(20.0)$ & $.51^{\mathrm{b}}$ \\
\hline Initial size of aneurysm (mean) (SD) ${ }^{a}$ & $7.7(3.3)$ & $7.2(3.2)$ & $10.2(3.5)$ & $.12^{\mathrm{a}}$ \\
\hline Aneurysm location (No.) (\%) & & & & $.70^{\mathrm{b}}$ \\
\hline Left VA & $18(34.6)$ & $25(44.6)$ & $1(20.0)$ & \\
\hline Right VA & $26(50)$ & $26(46.4)$ & $3(60.0)$ & \\
\hline Bilateral VA & $5(9.6)$ & $5(8.9)$ & $1(20.0)$ & \\
\hline BA & $2(3.8)$ & $0(0.0)$ & $0(0.0)$ & \\
\hline Both $V A$ and $B A$ & $1(1.9)$ & $0(0.0)$ & $0(0.0)$ & \\
\hline Radiologic changes & & & & $<.001^{\mathrm{b}}$ \\
\hline Improved (No.) (\%) & $1(1.9)$ & $15(26.8)$ & $1(20.0)$ & $<.001^{\mathrm{c}}$ \\
\hline Unchanged (No.) (\%) & $50(96.2)$ & $38(67.9)$ & $3(60.0)$ & $<.001^{\mathrm{d}}$ \\
\hline Enlarged (No.) (\%) & $1(1.9)$ & $3(5.4)$ & $1(20.0)$ & $.15^{\mathrm{e}}$ \\
\hline Clinical course & & & & $.048^{f}$ \\
\hline Stable (No.) (\%) & $51(98.1)$ & $55(98.2)$ & $4(80.0)$ & \\
\hline Deteriorated (No.) (\%) & $1(1.9)$ & $1(1.8)$ & $1(20.0)$ & \\
\hline
\end{tabular}

Note:-VA indicates vertebral artery; BA, basilar artery.

${ }^{a} P$ value was calculated by analysis of variance.

${ }^{b} P$ value was calculated by the $\chi^{2}$ test.

c $P$ value was calculated by the $\chi^{2}$ test between improved or not improved.

${ }^{d} P$ value was calculated by the $\chi^{2}$ test between unchanged or changed, either improved or enlarged.

e $P$ value was calculated by the $\chi^{2}$ test between enlarged or not enlarged.

${ }^{f} P$ value was calculated by the $\chi^{2}$ test between clinically stable or deteriorated.

\section{Statistical Analysis}

Associations between clinical presentation at diagnosis (asymptomatic, pain, and mass effects) and patient characteristics were evaluated by using the $\chi^{2}$ test and analysis of variance. Associations between reduction or enlargement in aneurysm size and clinical presentation at diagnosis and the size of aneurysms and other possible confounders (age, sex, hypertension, diabetes, hyperlipidemia, and smoking status) were evaluated by using the Cox proportional hazards model. Then, significant variables in the models were applied to a multivariate analysis. Two-sided $P$ values $<.05$ were considered statistically significant. All statistical analyses were performed by using STATA 12.0 (StataCorp, College Station, Texas).

\section{RESULTS}

Patient Characteristics

The final cohort consisted of 113 patients, ranging from 31 to 78 years of age
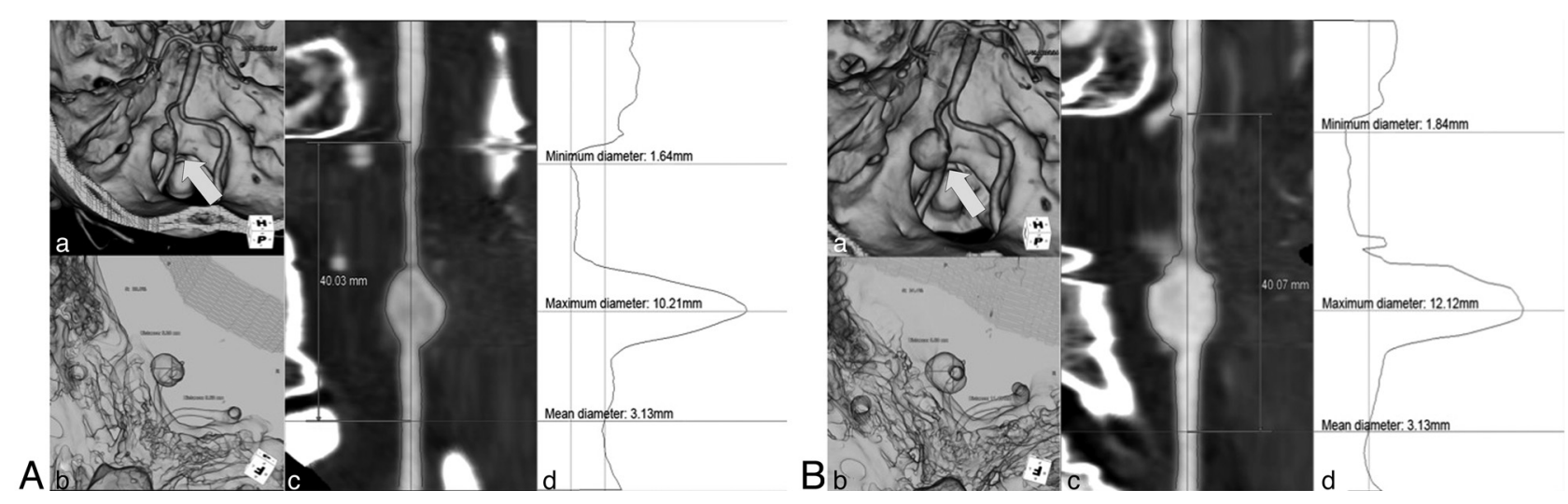

FIG 1. 3D-CTA reconstruction on the TeraRecon workstation and stretch view evaluating enlargement of the aneurysm diameter. Initial findings show a maximum diameter, $10.2 \mathrm{~mm}(A)$. Follow-up findings after 2 years show an increased maximum diameter, $12.2 \mathrm{~mm}(B)$. $a$, Volume rendering image (arrow indicates an aneurysm). b. Translucent image. c, MIP image of the stretch view. $d$, Calculated diameter. 
Table 2: Cases with radiologic and clinical deterioration

\begin{tabular}{cccccc}
\hline $\begin{array}{c}\text { Patient No. } \\
\text { (age }[\mathbf{y r}] \text { )/sex) }\end{array}$ & $\begin{array}{c}\text { Initial } \\
\text { Size }(\mathbf{m m})\end{array}$ & $\begin{array}{c}\text { Maximum } \\
\text { Size }(\mathbf{m m})\end{array}$ & $\begin{array}{c}\text { Follow-Up } \\
\text { Period }(\mathbf{m o})\end{array}$ & Clinical Deterioration & Treatment \\
\hline 48/M & 21.2 & 27.5 & 91.1 & Cerebellar ataxia & Endovascular trapping \\
59/F & 17.7 & 25.9 & 19.2 & Cerebellar ataxia, dysphagia & Endovascular trapping \\
43/F & 16.1 & 18.3 & 20.4 & Wallenberg syndrome, SAH & Endovascular trapping \\
35/M & 9.1 & 12.3 & 35.4 & None & Stent-assisted coiling \\
48/M & 10.1 & 12 & 8.3 & None & Stent-assisted coiling \\
\hline
\end{tabular}

Table 3: Cox proportional hazards model to find factors associated with aneurysm reduction

\begin{tabular}{|c|c|c|c|c|c|c|}
\hline & \multicolumn{3}{|c|}{$\begin{array}{c}\text { Single Cox Proportional } \\
\text { Hazards Model }\end{array}$} & \multicolumn{3}{|c|}{$\begin{array}{l}\text { Multiple Cox Proportional } \\
\text { Hazards Model }\end{array}$} \\
\hline & Hazard Ratio & $95 \% \mathrm{Cl}$ & $P$ Value & Hazard Ratio & $95 \% \mathrm{Cl}$ & $P$ Value \\
\hline Age (yr) & 0.93 & $0.89-0.98$ & .003 & 0.99 & $0.93-1.05$ & .63 \\
\hline Male sex & 0.68 & $0.25-1.86$ & .46 & & & \\
\hline \multicolumn{7}{|c|}{ Clinical presentation at diagnosis } \\
\hline Asymptomatic & Reference & - & - & Reference & - & - \\
\hline Pain & 14.7 & 1.94-111 & .009 & 3.63 & $0.38-34.8$ & .26 \\
\hline Mass effect & 7.94 & $0.50-127$ & .14 & 13.3 & $0.59-299$ & .10 \\
\hline \multicolumn{7}{|l|}{ Size } \\
\hline Maximum diameter (mm) & 0.61 & $0.46-0.82$ & .001 & 0.58 & $0.41-0.84$ & .004 \\
\hline$\geq 10 \mathrm{~mm}$ & 0.29 & $0.04-2.26$ & .24 & & & \\
\hline Hypertension & 0.08 & $0.02-0.35$ & .001 & 0.12 & $0.02-0.70$ & .018 \\
\hline \multicolumn{7}{|l|}{ Diabetes $^{b}$} \\
\hline Hyperlipidemia & 0.47 & $0.11-2.08$ & .32 & & & \\
\hline Smoking & 0.78 & $0.29-2.13$ & .63 & & & \\
\hline \multicolumn{7}{|l|}{ Location } \\
\hline Left & 1.39 & $0.16-12.5$ & .77 & & & \\
\hline Right & 2.87 & $0.37-22.1$ & .31 & & & \\
\hline Bilateral & Reference & & & & & \\
\hline
\end{tabular}

Note:- $\mathrm{Cl}$ indicates confidence interval.

${ }^{a}$ Variables that were statistically significant in single Cox proportional hazards models were used in the multiple Cox proportional hazards models. The cutoff point of the $P$ value was set at $<.05$.

${ }^{\mathrm{b}}$ All 6 patients with diabetes were excluded from this analysis because their aneurysms were unchanged.

(mean, 55 years) and 86 men (76\%). The mean follow-up was 2.9 years (range, 27 days to 8 years). Fifty-six (49.6\%) patients had a history of hypertension; 51 (45.1\%), smoking history; and 23 $(20.4 \%)$ a history of hyperlipidemia. Patients with hypertension and hyperlipidemia were on medication according to their physicians' prescriptions. Patients in group 1 were significantly older than those in group two: 59.3 years versus 51.1 years, respectively. Hypertension was more prevalent in group 1 than in the other 2 groups.

\section{Clinical Findings}

Of 113 patients, 3 deteriorated during the study period, and the incidence of clinical deterioration was significantly higher in group $3(P=.048)$. One patient in group $3(0.9 \%)$ developed an ischemic stroke (Wallenberg syndrome) and was admitted to another institution where the patient received anticoagulation therapy. Subsequently, the patient had SAH (Hunt and Hess grade 2) and was transferred back to our institution, where endovascular obliteration was performed. Two patients (1.8\%), 1 in group 1 and 1 in group 2, experienced new clinical symptoms due to mass effect (Tables 1 and 2).

\section{Radiologic Findings}

Radiologic evaluation of aneurysm size revealed a statistically significant difference $(P<.001)$ among the 3 groups: In most of the patients in group 1 (96.2\%), aneurysm size remained unchanged, in contrast to $26.8 \%$ of patients in group $2 \%$ and $20 \%$ in group 3 in whom aneurysm size became smaller.
On the basis of proportional hazards models, pain as a clinical presentation at diagnosis significantly increased the chance for aneurysm reduction. In contrast, larger maximum aneurysm diameter and hypertension at diagnosis were associated with less possibility of aneurysm reduction. Using an adjustment with significant variables, a multiple regression analysis found that pain and aneurysm size at diagnosis were still significantly associated with aneurysm reduction (Table 3 ).

Next, similar to our analysis of the factors associated with aneurysm reduction, we analyzed the factors associated with aneurysm enlargement. The risk of aneurysm enlargement was significantly associated with either maximum diameter (hazard ratio $=$ 1.30; 95\% CI, 1.11-11.52; $P=.001)$ or aneurysm of $\geq 10 \mathrm{~mm}$ (hazard ratio $=18.0 ; 95 \% \mathrm{CI}, 1.95-167 ; P=.011)$ (Table 4). All 3 patients who clinically deteriorated showed enlarged aneurysms, and that association was statistically significant $(P<.001)$, though the number was small.

\section{DISCUSSION}

\section{Management Strategy and Clinical Findings}

Reports of incidentally found DVBAs are rare, but the detection of DVBAs is rising due to increased and improved screening by brain imaging. Therapeutic management of patients with DVBA who are asymptomatic or present only with pain remains controversial and is not well-established. ${ }^{2,3}$ DVBA with pain is considered a critical condition predictive of stroke, for which either a surgical or endovascular 
Table 4: Cox proportional hazards model to find factors associated with aneurysm enlargement

\begin{tabular}{|c|c|c|c|}
\hline & \multicolumn{3}{|c|}{$\begin{array}{c}\text { Single Cox Proportional } \\
\text { Hazards Model }\end{array}$} \\
\hline & Hazard Ratio & $95 \% \mathrm{Cl}$ & $P$ Value \\
\hline Age $(y r)$ & 0.93 & $0.85-1.01$ & .086 \\
\hline Male sex & 0.54 & $0.09-3.22$ & .50 \\
\hline \multicolumn{4}{|l|}{$\begin{array}{c}\text { Clinical presentation at } \\
\text { diagnosis }\end{array}$} \\
\hline Asymptomatic & Reference & - & - \\
\hline Pain & 2.83 & $0.29-27.3$ & .37 \\
\hline Mass effect & 9.38 & $0.56-156$ & .12 \\
\hline \multicolumn{4}{|l|}{ Size } \\
\hline Maximum diameter (mm) & 1.30 & $1.11-1.52$ & .001 \\
\hline$\geq 10 \mathrm{~mm}$ & 18.0 & $1.95-167$ & .011 \\
\hline Hypertension & 0.38 & $0.06-2.34$ & .30 \\
\hline \multicolumn{4}{|l|}{ Diabetes $^{a}$} \\
\hline Hyperlipidemia & 0.88 & $0.10-7.92$ & .91 \\
\hline Smoking & 0.85 & $0.14-5.10$ & .86 \\
\hline \multicolumn{4}{|l|}{ Location } \\
\hline Left & 0.13 & $0.01-1.65$ & .12 \\
\hline Right & 0.19 & $0.02-1.57$ & .12 \\
\hline Bilateral & Reference & & \\
\hline
\end{tabular}

Note:- $\mathrm{Cl}$ indicates confidence interval.

a All 6 patients with diabetes were excluded from this analysis because their aneurysms were unchanged.

approach is the treatment of choice. ${ }^{4-6}$ Rabinov et $\mathrm{al}^{7}$ reported on 28 patients with DVBA who were treated surgically or endovascularly; 2 patients presented with pain only, of whom 1 was surgically treated and rated a score of 3 on the modified Rankin Scale. Ahn et $\mathrm{al}^{5}$ reported their endovascular treatment of 14 patients with DVBA, of whom 5 presented with headache only. In our series, none of the patients who presented with pain only at diagnosis had a hemorrhagic or ischemic stroke during observation. On the basis of our results, aggressive interventional treatment may not be necessary for patients with DVBA presenting with pain only.

The establishment of optimal medical management is also controversial. Kim et $\mathrm{al}^{8}$ reported their large series of 191 patients with unruptured vertebrobasilar artery dissections, including ischemic and nonischemic symptoms, in which 81 patients presented with headache only. All were treated either endovasculary ( $n=46)$ or by medical therapy with anticoagulation $(n=49)$, antiplatelet therapy $(n=48)$, or analgesics $(n=48)$. The necessity for anticoagulation or antiplatelet therapy remains unclear; our results, at least, do not prove the necessity for anticoagulation or antiplatelet therapy for patients presenting with pain only.

We did not use any antiplatelet or anticoagulation therapy during observation. One patient who presented with mass effect at diagnosis had a brain stem infarction during observation and was admitted to another hospital. The patient received anticoagulation therapy and subsequently experienced SAH. Some similarities can be found with Tsutsumi et al, ${ }^{9}$ who reported a vertebral artery dissection causing SAH in a similar patient who had initially presented with infarction and had received antiplatelet agent therapy. Thus, pain management and blood pressure control are recommended for patients presenting with pain only or ischemic symptoms.

Yasui et $\mathrm{al}^{10}$ reported their histopathologic findings of incidentally detected fusiform vertebrobasilar aneurysms, which demonstrated intimal thickening, disruption of the internal elastic lamina, and degeneration of the media. They concluded that an incidental fusiform vertebrobasilar aneurysm has the potential to develop into a dissecting aneurysm. In our series, only 1 patient with an incidentally found DVBA experienced clinical deterioration and aneurysm growth during observation.

\section{Morphologic Changes}

Observation with serial radiologic examinations should be performed on patients with unruptured DVBAs. Most of the DVBAs in group 1 did not show morphologic findings in the vessel wall. Sato et $\mathrm{al}^{11}$ showed that disrupted internal elastic lamina, covered with intimal thickening, is commonly found at postmortem examination in normal intracranial vertebral arteries of patients who died of causes other than intracranial lesions. Most of the incidentally detected lesions occurred silently or with minor headache and vessel wall healing occurred silently.

Ahn et $\mathrm{al}^{12}$ reported the difference in the morphologic evolution of the lesion; 25 of 34 symptomatic intracranial vertebrobasilar dissections with dilation without stenosis had no change compared with their initial shape. Pozzati et al ${ }^{13}$ described cases of spontaneous resolution of fusiform aneurysm in the vertebrobasilar system proved by angiography. Naito et $\mathrm{al}^{1}$ reported that angiographic features of vertebral artery dissection changed for 12 of 20 patients $(60 \%)$ and that deterioration of features was seen in 4 cases $(20 \%)$. Nakagawa et $\mathrm{al}^{14}$ reported serial angiographic changes in $88.2 \%$ of unruptured vertebral artery dissections. In our series, morphologic changes were observed in 22 of 113 patients $(19.5 \%)$ and $5(4.4 \%)$ had DVBAs that enlarged. As for DVBAs having the possibility of changing morphologically, we suggest serial radiologic follow-up for nonstroke DVBAs.

Mizutani ${ }^{15}$ reported on 44 patients who presented with nonischemic unruptured dissections in the vertebrobasilar system, of which 10 DVBAs improved, 4 enlarged, and 1 ruptured during observation. Mangrum et $\mathrm{al}^{16}$ reported that enlargement of nonsaccular intracranial aneurysms correlated significantly with median aneurysm diameter and symptomatic compression at diagnosis. Flemming et $\mathrm{al}^{17}$ reported that the annual prospective risk of hemorrhage from a vertebrobasilar artery nonsaccular intracranial aneurysm is $0.9 \%$ and that an aneurysm diameter of at least $10 \mathrm{~mm}$ is strongly indicative of future rupture. In our series, aneurysms of $<10 \mathrm{~mm}$ had a favorable clinical outcome, but aneurysms of $>10 \mathrm{~mm}$ with symptoms due to mass effect had a risk of clinical deterioration and enlargement. In such cases, surgical or endovascular intervention should be considered.

\section{Limitations of the Study}

This study has several limitations. First, the radiologic examination findings were not the same for all patients. The diameter of the aneurysm was different between CTA and MRA. Nevertheless, the goal of this study was not to compare the differences in the devices but to evaluate the changes in findings with the same method. Each patient was evaluated with either CTA or MRA TOF only. Second, we defined "DVBA" as a nonsaccular aneurysm located at a nonbranching site of the vertebrobasilar artery; however, it is sometimes difficult to determine whether an incidental asymptomatic fusiform aneurysm may have resulted from spontaneously healed dissections or other underlying vascular abnormalities, especially with only CT. Third, intramural hema- 
toma was significantly associated with a change of vertebrobasilar dissection on follow-up in a previous report. ${ }^{12}$ In this study, most imaging was performed with CT angiography. CTA has limitations in detecting an intramural hematoma. Fourth, in this study, mean follow-up was 2.9 years. This time is relatively short to evaluate the natural history of these lesions. In this study group, 3 patients demonstrated relatively good clinical outcome, though these patients showed significant risk of enlargement. This discrepancy may be due to lack of long-term follow-up. Fifth, in some cases, follow-up was discontinued, and these patients might have experienced SAH or infarction and might have been admitted elsewhere. Finally, we have a small number of patients with nonstroke DVBA who were treated with endovascular therapy; they do not entirely reflect the results of the study, but this small number will have a negligible effect on the natural course of nonstroke DVBA for the whole group. Although the study has these limitations, the results may provide important information for the treatment and further investigation of nonstroke DVBA.

\section{CONCLUSIONS}

The natural course of nonstroke DVBA is favorable during a 2.9year period. The short-term course of these lesions suggests that acute intervention is not always required and close follow-up is reasonable, unless patients develop symptoms associated with significant mass effect. Patients with symptoms due to mass effect or the size of the aneurysm (diameter of $>10 \mathrm{~mm}$ ) may deteriorate and eventually require intervention.

\section{ACKNOWLEDGMENTS}

The authors acknowledge the assistance of Naoya Kunikane, RT, Yukiko Abe, RT, and Hiroto Narita, PhD, with technical support for 3D-CTA.

\begin{abstract}
Disclosures: Yuichi Murayama—UNRELATED: Consultancy: Asahi Intecc, Stryker, Grants/Grants Pending: Siemens, ${ }^{*}$ Stryker, ${ }^{\star}$ Asahi Intecc, ${ }^{*}$ Fujifilm, ${ }^{\star}$ Patents (planned, pending, or issued): Stryker, ${ }^{\star}$ Royalties: Stryker.* Ichiro Yuki-UNRELATED: Grants/ Grants Pending: Siemens. * Toshihiro Ishibashi-UNRELATED: Consultancy: Stryker Japan. Hiroyuki Takao—UNRELATED: Grants/Grants Pending: Fujifilm, ${ }^{*}$ NTT DoCoMo.* Ikki Kajiwara—UNRELATED: Grants/Grants Pending: Siemens.* Mitsuyoshi UrashimaUNRELATED: Grants/Grants Pending: Ministry of Education, Culture, Sports, Science, and Technology in Japan, a supported program for the Strategic Research Foundation at private universities. * * Money paid to the institution.
\end{abstract}

\section{REFERENCES}

1. Naito I, Iwai T, Sasaki T. Management of intracranial vertebral artery dissections initially presenting without subarachnoid hemorrhage. Neurosurgery 2002;51:930-37, discussion 937-38
2. Kai Y, Nishi T, Watanabe M, et al. Strategy for treating unruptured vertebral artery dissecting aneurysms. Neurosurgery 2011;69:108591, discussion 1091-92

3. Santos-Franco JA, Zenteno M, Lee A. Dissecting aneurysms of the vertebrobasilar system: a comprehensive review on natural history and treatment options. Neurosurg Rev 2008;31:131-40, discussion 140

4. Jin SC, Kwon DH, Choi CG, et al. Endovascular strategies for vertebrobasilar dissecting aneurysms. AJNR Am J Neuroradiol 2009;30:1518-23

5. Ahn JY, Han IB, Kim TG, et al. Endovascular treatment of intracranial vertebral artery dissections with stent placement or stent-assisted coiling. AJNR Am J Neuroradiol 2006;27:1514-20

6. Shin YS, Kim HS, Kim SY. Stenting for vertebrobasilar dissection: a possible treatment option for nonhemorrhagic vertebrobasilar dissection. Neuroradiology 2007;49:149-56

7. Rabinov JD, Hellinger FR, Morris PP, et al. Endovascular management of vertebrobasilar dissecting aneurysms. AJNR Am J Neuroradiol 2003;24:1421-28

8. Kim BM, Kim SH, Kim DI, et al. Outcomes and prognostic factors of intracranial unruptured vertebrobasilar artery dissection. Neurology 2011;76:1735-41

9. Tsutsumi M, Kawano T, Kawaguchi T, et al. Dissecting aneurysm of the vertebral artery causing subarachnoid hemorrhage after nonhemorrhagic infarction: case report. Neurol Med Chir (Tokyo) 2000;40:628-31

10. Yasui T, Komiyama M, Iwai Y, et al. Evolution of incidentally-discovered fusiform aneurysms of the vertebrobasilar arterial system: neuroimaging features suggesting progressive aneurysm growth. Neurol Med Chir (Tokyo) 2001;41:523-27, discussion 528

11. Sato T, Sasaki T, Suzuki K, et al. Histological study of the normal vertebral artery: etiology of dissecting aneurysms. Neurol Med Chir (Tokyo) 2004;44:629-35, discussion 636

12. Ahn SS, Kim BS, Suh SH, et al. Spontaneous symptomatic intracranial vertebrobasilar dissection: initial and follow-up imaging findings. Radiology 2012;264:196-202

13. Pozzati E, Padovani R, Fabrizi A, et al. Benign arterial dissections of the posterior circulation. J Neurosurg 1991;75:69-72

14. Nakagawa K, Touho H, Morisako T, et al. Long-term follow-up study of unruptured vertebral artery dissection: clinical outcomes and serial angiographic findings. J Neurosurg 2000; 93:19-25

15. Mizutani T. Natural course of intracranial arterial dissections. J Neurosurg 2011;114:1037-44

16. Mangrum WI, Huston J 3rd, Link MJ, et al. Enlarging vertebrobasilar nonsaccular intracranial aneurysms: frequency, predictors, and clinical outcome of growth. J Neurosurg 2005;102:72-79

17. Flemming KD, Wiebers DO, Brown RD Jr, et al. Prospective risk of hemorrhage in patients with vertebrobasilar nonsaccular intracranial aneurysm. J Neurosurg 2004;101:82-87 